\title{
Mingling: mixed-integer rounding with bounds
}

\author{
Alper Atamtürk • Oktay Günlük
}

Received: 24 January 2008 / Accepted: 28 July 2008 / Published online: 31 January 2009

C The Author(s) 2009. This article is published with open access at Springerlink.com

\begin{abstract}
Mixed-integer rounding (MIR) is a simple, yet powerful procedure for generating valid inequalities for mixed-integer programs. When used as cutting planes, MIR inequalities are very effective for mixed-integer programming problems with unbounded integer variables. For problems with bounded integer variables, however, cutting planes based on lifting techniques appear to be more effective. This is not surprising as lifting techniques make explicit use of the bounds on variables, whereas the MIR procedure does not. In this paper we describe a simple procedure, which we call mingling, for incorporating variable bound information into MIR. By explicitly using the variable bounds, the mingling procedure leads to strong inequalities for mixed-integer sets with bounded variables. We show that facets of mixed-integer knapsack sets derived earlier by superadditive lifting techniques can be obtained by the mingling procedure. In particular, the mingling inequalities developed in this paper subsume the continuous cover and reverse continuous cover inequalities of Marchand and Wolsey (Math Program 85:15-33, 1999) as well as the continuous integer knapsack cover and pack inequalities of Atamtürk (Math Program 98:145-175, 2003; Ann Oper Res 139:21-38, 2005). In addition, mingling inequalities give a generalization of the
\end{abstract}

This research is funded, in part, by an IBM faculty award. A. Atamtürk is grateful for the hospitality of the Georgia Institute of Technology, where part of this research was conducted.

\footnotetext{
A. Atamtürk $(\varangle)$

Department of Industrial Engineering and Operations Research,

University of California, Berkeley, CA 94720-1777, USA

e-mail: atamturk@berkeley.edu

O. Günlük

Mathematical Sciences Department, IBM T. J. Watson Research Center,

Yorktown Heights, NY 10598, USA

e-mail: gunluk@us.ibm.com
} 
two-step MIR inequalities of Dash and Günlük (Math Program 105:29-53, 2006) under some conditions.

Keywords Cutting planes $\cdot$ Mixed-integer rounding $\cdot$ Superadditive lifting

Mathematics Subject Classification (2000) $90 \mathrm{C} 11$

\section{Introduction}

Mixed-integer rounding (MIR) is a general procedure for deriving valid inequalities for mixed-integer programming. The MIR cuts, introduced by Nemhauser and Wolsey $[11,12]$, are applied to a single constraint (possibly implied by other constraints) of a mixed-integer program (MIP), much like the Chvátal-Gomory integer rounding cuts [5] for pure integer programs. MIR cuts are equivalent to Gomory's mixed-integer cuts for MIPs [8] and split cuts of Cook et al. [6] and are a special case of the disjunctive cuts of Balas [4].

Marchand and Wolsey [10] show that when applied carefully MIR cuts can give some of the well-known strong cuts for special mixed-integer sets. In their computational study, they make a convincing case that applying MIR cuts after aggregating constraints into a single constraint and complementing variables - that is, replacing a variable $x$ satisfying $0 \leq x \leq u$ with $\bar{x}=u-x$ by appropriately updating the coefficients of the constraint-is very effective in solving MIPs. MIR cuts are implemented in major MIP solvers, more or less, by following this approach.

Unlike lifted inequalities for sets with special structures, the MIR cuts do not explicitly use the upper bounds of the variables; however, they use their lower bounds. Therefore, complementing variables allows MIR to make use of the upper bound information to some degree. In this paper we present a new way of incorporating upper bounds of the variables by a simple procedure, which we refer to as mingling the variables. By explicitly using the variable bounds, the mingling procedure leads to strong inequalities for mixed-integer sets with bounded variables. We show that facets of the mixed-integer knapsack sets derived earlier by superadditive lifting techniques are, indeed, mingling inequalities or two-step mingling inequalities.

Let us now recall the MIR inequalities. Consider the mixed-integer set given by

$$
\sum_{i \in N} a_{i} x_{i}+s \geq b, \quad x \in \mathbb{Z}_{+}^{N}, s \in \mathbb{R}_{+},
$$

where the base inequality $a x+s \geq b$ may be implied by constraints of an MIP. For any $\alpha>0$, the $\alpha$-MIR inequality for (1) is

$$
\sum_{i \in N} \mu_{\alpha, b}\left(a_{i}\right) x_{i}+s \geq \mu_{\alpha, b}(b),
$$

where

$$
\begin{aligned}
\mu_{\alpha, b}\left(a_{i}\right) & =r\left\lfloor a_{i} / \alpha\right\rfloor+\min \left\{r, r_{i}\right\}, \quad i \in N, \\
\mu_{\alpha, b}(b) & =r\lfloor b / \alpha\rfloor+r,
\end{aligned}
$$


and

$$
r=b-\alpha\lfloor b / \alpha\rfloor, \quad r_{i}=a_{i}-\alpha\left\lfloor a_{i} / \alpha\right\rfloor, \quad i \in N
$$

Observe that an $\alpha$-MIR inequality is the 1-MIR inequality written after dividing the base inequality by $\alpha>0$. In order to highlight the inequalities of this paper, it is important to remark that nonnegativity of $x_{i}, i \in N$, is necessary for the validity of the $\alpha$-MIR inequality unless $a_{i} / \alpha \in \mathbb{Z}$ (see Marchand and Wolsey [10] for a simple proof of validity of the MIR inequalities).

Lemma 1 [11] The MIR function $\mu_{\alpha, b}$ is nondecreasing and subadditive for $\alpha>0$.

If it is known that $a, b \geq 0$, then using the nonnegativity of the variables, we can first strengthen the base inequality as

$$
\sum_{i \in N} \min \left\{a_{i}, b\right\} x_{i}+s \geq b
$$

and then apply $\alpha$-MIR to obtain

$$
\sum_{i \in N} \mu_{\alpha, b}\left(\min \left\{a_{i}, b\right\}\right) x_{i}+s \geq \mu_{\alpha, b}(b),
$$

which dominates $\alpha$-MIR inequality (2) as $\mu_{\alpha, b}$ is nondecreasing.

In this paper, we present a similar strengthening idea when the coefficients of the base inequality are unrestricted in sign by using the lower bounds as well as the upper bounds of the variables. We illustrate this point with a simple set in the next section. In Sects. 3 and 4 we present the mingling inequalities in general form and in Sect. 5 we show the connection of the mingling inequalities with other inequalities given in the literature before. Finally, in Sect. 6 we conclude with a few closing remarks.

\section{A simple set}

In this section, we describe a simple mixed-integer knapsack set to give an intuition for the inequalities that incorporate variable bound information into MIR. Consider the following three-variable set

$$
S=\left\{(x, s) \in \mathbb{Z}^{2} \times \mathbb{R}: a_{1} x_{1}+x_{2}+s \geq b, x_{1} \geq 0, u_{2} \geq x_{2} \geq 0, s \geq 0\right\},
$$

where the coefficients satisfy $a_{1}<0<b<1 \leq u_{2}$ and $u_{2} \in \mathbb{Z}$. Because $a_{1}<0$, coefficient improvement as in (3) is not applicable to the base inequality $a_{1} x_{1}+x_{2}+s \geq b$. However, in this case, we can utilize the upper bound of $x_{2}$ in order to derive a valid inequality that generalizes the MIR inequalities. 
2.1 Basic inequality

Adding and subtracting $u_{2} x_{1}$, we rewrite the base inequality of $S$ as

$$
\left(a_{1}+u_{2}\right) x_{1}+\left(x_{2}-u_{2} x_{1}\right)+s \geq b .
$$

By considering the disjunction $x_{1}=0 \vee x_{1} \geq 1$, we obtain from (5) the valid inequality

$$
\left(a_{1}+u_{2}\right) x_{1}+b\left(x_{2}-u_{2} x_{1}\right)+s \geq b
$$

for $S$. For $x_{1}=0$, inequality (6) is the 1-MIR inequality for (5) as $0<b<1$ and $s \geq 0$; and for $x_{1} \geq 1$, inequality (6) is a relaxation of (5) as $b<1$ and $x_{2}-u_{2} x_{1} \leq 0$. This type of coefficient improvement using the upper bound of $x_{2}$ is of interest when $a_{1}+u_{2}<0$ because otherwise, the 1-MIR inequality

$$
\mu_{1, b}\left(a_{1}\right) x_{1}+\mu_{1, b}(1) x_{2}+s \geq \mu_{1, b}(b)
$$

is at least as strong as (6). Writing (7) explicitly as

$$
\min \left\{a_{1}-\left\lfloor a_{1}\right\rfloor, b\right\} x_{1}+b\left(x_{2}+\left\lfloor a_{1}\right\rfloor x_{1}\right)+s \geq b
$$

makes the comparison easier. Now, defining $k:=\min \left\{u_{2},-\left\lfloor a_{1}\right\rfloor\right\}$, we can generalize (6) and (8) as

$$
\min \left\{a_{1}+k, b\right\} x_{1}+b\left(x_{2}-k x_{1}\right)+s \geq b .
$$

Observe that if $u_{2}<-\left\lfloor a_{1}\right\rfloor$, then (9) is stronger than 1-MIR inequality (8). Otherwise, (9) is at least as strong as (6). Indeed, inequality (9) is facet-defining for $\operatorname{conv}(S)$, which is easily checked with the affinely independent points $\left(x_{1}, x_{2}, s\right)$ of $S$ listed below:

$$
(0,0, b), \quad(0,1,0), \quad\left(1, k,\left(b-a_{1}-k\right)^{+}\right) .
$$

Hence, by using the upper bound of $x_{2}$, we have strengthened the basic MIR inequality (7). In Sect. 3, we generalize inequality (9) to obtain the mingling inequality (21).

Remark 1 We should point out that complementing $x_{2}$ in the base inequality and then applying 1-MIR does not lead to a new inequality as

$$
\mu_{1, b}\left(a_{1}\right) x_{1}+\mu_{1, b}(-1)\left(u_{2}-x_{2}\right)+s \geq \mu_{1, b}\left(b-u_{2}\right)
$$

which equals

$$
\mu_{1, b}\left(a_{1}\right) x_{1}-b\left(u_{2}-x_{2}\right)+s \geq b-b u_{2}
$$

for any $u_{2} \in \mathbb{Z}$, is the 1 -MIR inequality (7) obtained without complementing $x_{2}$. 


\subsection{A two-step inequality}

Next we will derive a new inequality based on (9). First we consider the case $a_{1}+k<b$. As inequality (9) and

$$
\left(a_{1}+k\right) x_{1}+\left(x_{2}-k x_{1}\right)+s \geq b
$$

are both valid for $S$, their convex combination

$$
\left(a_{1}+k\right) x_{1}+\beta\left(x_{2}-k x_{1}\right)+s \geq b,
$$

where $b \leq \beta \leq 1$, is valid as well. Choosing $\beta=\alpha\lceil b / \alpha\rceil \leq 1$ for some $\alpha>0$ such that $b / \alpha \notin \mathbb{Z}$ and applying $\alpha$-MIR to (10), we obtain

$$
\mu_{\alpha, b}\left(a_{1}+k\right) x_{1}+\mu_{\alpha, b}(\alpha\lceil b / \alpha\rceil)\left(x_{2}-k x_{1}\right)+s \geq \mu_{\alpha, b}(b),
$$

or, equivalently,

$$
\mu_{\alpha, b}\left(a_{1}+k\right) x_{1}+\mu_{\alpha, b}(b)\left(x_{2}-k x_{1}\right)+s \geq \mu_{\alpha, b}(b) .
$$

Note that validity of the $\alpha$-MIR inequality (11) crucially depends on the fact that $\beta / \alpha$ is integral since $x_{2}-k x_{1}$ may not be nonnegative.

Next consider the case $a_{1}+k \geq b$. We now write the base inequality as

$$
\left(a_{1}+(k-1)\right) x_{1}+\left(x_{2}-(k-1) x_{1}\right)+s \geq b,
$$

which, as $a_{1}+(k-1) \leq 0$ in this case, can be relaxed to

$$
1\left(x_{2}-(k-1) x_{1}\right)+s \geq b .
$$

Also inequality (9) can be written in a similar form as

$$
b\left(x_{2}-(k-1) x_{1}\right)+s \geq b .
$$

Then,

$$
\beta\left(x_{2}-(k-1) x_{1}\right)+s \geq b,
$$

where $b \leq \beta \leq 1$, is valid as well. Choosing $\beta=\alpha\lceil b / \alpha\rceil \leq 1$ for some $\alpha>0$ such that $b / \alpha \notin \mathbb{Z}$ and applying $\alpha$-MIR to (13), this time, we obtain

$$
\mu_{\alpha, b}(b)\left(x_{2}-(k-1) x_{1}\right)+s \geq \mu_{\alpha, b}(b) .
$$

Combining (12) and (14), we obtain the following valid inequality for $S$ :

$$
\mu_{\alpha, b}\left(\min \left\{a_{1}+k, b\right\}\right) x_{1}+\mu_{\alpha, b}(b)\left(x_{2}-k x_{1}\right)+s \geq \mu_{\alpha, b}(b) .
$$


We note that when $u_{2} \geq-\left\lfloor a_{1}\right\rfloor$, inequality (15) becomes the two-step MIR inequality [7]. In Sect. 3, we generalize inequality (15) to obtain the two-step mingling inequality (31).

Remark 2 It is of interest to know how inequality (15) compares with a direct application of MIR to inequality (9). In order to do so, we collect the terms for $x_{1}$ in (9) and rewrite it as

$$
\left(\min \left\{a_{1}+k, b\right\}-b k\right) x_{1}+b x_{2}+s \geq b .
$$

Applying $\alpha$-MIR to this inequality, we obtain

$$
\mu_{\alpha, b}\left(\min \left\{a_{1}+k, b\right\}-b k\right) x_{1}+\mu_{\alpha, b}(b) x_{2}+s \geq \mu_{\alpha, b}(b) .
$$

If $\alpha$ is chosen as above, then the difference between (15) and (16) is only the coefficient of $x_{1}$. However, because $\mu_{\alpha, b}$ is subadditive and $k \in \mathbb{Z}_{+}$, we have

$$
\begin{aligned}
\mu_{\alpha, b}\left(\min \left\{a_{1}+k, b\right\}\right) & \leq \mu_{\alpha, b}\left(\min \left\{a_{1}+k, b\right\}-b k\right)+\mu_{\alpha, b}(b k) \\
& \leq \mu_{\alpha, b}\left(\min \left\{a_{1}+k, b\right\}-b k\right)+k \mu_{\alpha, b}(b)
\end{aligned}
$$

and, therefore, (15) is at least as strong as (16). The numerical example below illustrates that (15) dominates (16) strictly.

Example 1 Let set $S$ be given as

$$
-5 x_{1}+x_{2}+s \geq 0.5, \quad s \geq 0, \quad x_{1} \geq 0, \quad 2 \geq x_{2} \geq 0 .
$$

Then $k=\min \{2,5\}=2$ and inequality (9) is

$$
-3 x_{1}+0.5\left(x_{2}-2 x_{1}\right)+s \geq 0.5 \text {. }
$$

For $\alpha=0.3$, we have $r=0.2$, and inequality (15)

$$
-2 x_{1}+0.4\left(x_{2}-2 x_{1}\right)+s \geq 0.4
$$

strictly dominates inequality (16)

$$
-2.6 x_{1}+0.4 x_{2}+s \geq 0.4 \text {. }
$$

We note that this inequality is not facet-defining for $\operatorname{conv}(S)$. 


\section{A mingling procedure}

In this section we generalize inequalities (9) and (15) to obtain valid inequalities for the mixed-integer knapsack set

$$
K_{\geq}:=\left\{(x, s) \in \mathbb{Z}^{N} \times \mathbb{R}: \sum_{i \in I} a_{i} x_{i}+\sum_{j \in J} a_{j} x_{j}+s \geq b, u \geq x \geq 0, s \geq 0\right\}
$$

where $\max _{i \in I}\left\{a_{i}\right\}>b$ and $(I, J)$ is the partitioning of $N$ with $a_{i}>0$ for $i \in I$ and $a_{j}<0$ for $j \in J$. We allow the upper bound on each variable to be either a positive integer or infinite. Throughout this section we assume that $b \geq 0$ and derive valid inequalities for $K_{\geq}$using upper bounds $u_{i}, i \in I$. In the next section, we derive valid inequalities when $b \leq 0$ using upper bounds $u_{j}, j \in J$.

\subsection{Mingling inequalities}

Let us first introduce some new notation. For $x \in \mathbb{Z}^{N}$, let $x(S):=\sum_{i \in S} x_{i}$ for $S \subseteq N$. Let $I^{+}:=\{1, \ldots, n\}$ be a nonempty subset of $\left\{i \in I: a_{i}>b\right\}$ indexed in nonincreasing order of $a_{i}$ 's, $\kappa:=\sum_{i \in I^{+}} a_{i} u_{i}$, and $\bar{J}:=\left\{j \in J: a_{j}+\kappa<0\right\}$.

For any $j \in J \backslash \bar{J}$, we next define a set $I_{j}$ and numbers $0 \leq \bar{u}_{i j} \leq u_{i}$ for $i \in I_{j}$ such that $a_{j}+\sum_{i \in I_{j}} a_{i} \bar{u}_{i j} \geq 0$. More precisely, for $j \in J \backslash \bar{J}$, let

$$
I_{j}:=\{1, \ldots, p(j)\}, \quad \text { where } p(j):=\min \left\{p \in I^{+}: a_{j}+\sum_{i=1}^{p} a_{i} u_{i} \geq 0\right\}
$$

and

$$
k_{j}:=\min \left\{k \in \mathbb{Z}_{+}: a_{j}+\sum_{i=1}^{p(j)-1} a_{i} u_{i}+a_{p(j)} k \geq 0\right\}
$$

Furthermore, for $j \in J \backslash \bar{J}$, and $i \in I_{j}$, let

$$
\bar{u}_{i j}= \begin{cases}u_{i}, & \text { if } \quad i<p(j) \\ k_{j}, & \text { if } i=p(j)\end{cases}
$$

For $j \in \bar{J}$, we let $I_{j}:=I^{+}, p(j):=n, k_{j}:=u_{n}$ and $\bar{u}_{i j}=u_{i}$ for $i \in I_{j}$.

For $i \in I$, let $J_{i}:=\left\{j \in J: i \in I_{j}\right\}$; hence, $J_{i}=\emptyset$ for $i \in I \backslash I^{+}$. Observe that the definitions of mingling sets $I_{j}$ and $J_{i}$ imply that they are nested. Precisely,

$$
\text { for } i, k \in I^{+}, a_{i}>a_{k} \Rightarrow J_{k} \subseteq J_{i}
$$


and

$$
\text { for } j, k \in J, \quad a_{j}<a_{k} \Rightarrow I_{k} \subseteq I_{j} \text {. }
$$

The nestedness property of the mingling sets is crucial for the validity of the mingling inequalities introduced next.

Using the mingling sets defined above we can now write the base inequality in $K_{\geq}$ as follows:

$$
\sum_{i \in I} a_{i}\left(x_{i}-\sum_{j \in J_{i}} \bar{u}_{i j} x_{j}\right)+\sum_{j \in J}\left(a_{j}+\sum_{i \in I_{j}} a_{i} \bar{u}_{i j}\right) x_{j}+s \geq b .
$$

Note that in this form $a_{j}+\sum_{i \in I_{j}} a_{i} \bar{u}_{i j}$ is nonnegative for $j \in J \backslash \bar{J}$ and negative for $j \in \bar{J}$. The main result of this section is the derivation of the mingling inequality

$$
\sum_{i \in I^{+}} b\left(x_{i}-\sum_{j \in J_{i}} \bar{u}_{i j} x_{j}\right)+\sum_{i \in I \backslash I^{+}} a_{i} x_{i}+\sum_{j \in J} \min \left\{b, a_{j}+\sum_{i \in I_{j}} a_{i} \bar{u}_{i j}\right\} x_{j}+s \geq b
$$

from (20). Observe that if $I^{+}=\emptyset$, then inequality (21) reduces to the base inequality (20). The validity of the mingling inequality for $K_{\geq}$is not obvious and does not follow from MIR of (20) because the terms $\left(x_{i}-\sum_{j \in J_{i}} \bar{u}_{i j} x_{j}\right), i \in I^{+}$, and $\left(a_{j}+\sum_{i \in I_{j}} a_{i} \bar{u}_{i j}\right), j \in J$, are not necessarily nonnegative. Furthermore, it does not seem to be possible to derive inequality (21) as a straightforward extension of (9). In the following, we first prove the validity and then the strength of the mingling inequality.

Proposition 1 The mingling inequality (21) is valid for $K_{\geq}$.

Proof First we will write inequality (21) more explicitly with the aid of some new notation. For $j \in J$, let

$$
\delta_{j}=a_{j}+\sum_{i=1}^{p(j)-1} a_{i} u_{i}+a_{p(j)} k_{j}=a_{j}+\sum_{i \in I_{j}} a_{i} \bar{u}_{i j}
$$

We have $\delta_{j}<0$ for $j \in \bar{J}$ and $\delta_{j} \geq 0$ for $j \in J \backslash \bar{J}$. For $j \in J \backslash \bar{J}$ and $i \in I_{j}$, let

$$
\tilde{u}_{i j}= \begin{cases}u_{i}, & \text { if } i<p(j), \\ k_{j}, & \text { if } i=p(j) \quad \text { and } \quad \delta_{j}<b \\ k_{j}-1, & \text { if } i=p(j) \quad \text { and } \quad \delta_{j} \geq b\end{cases}
$$


Note that $\tilde{u}_{i j}=\bar{u}_{i j}-1$ if $i=p(j)$ and $\delta_{j} \geq b$, and $\tilde{u}_{i j}=\bar{u}_{i j}$ otherwise. Also observe that for $j \in J \backslash \bar{J}$

$$
a_{j}+\sum_{i \in I_{j}} a_{i} \tilde{u}_{i j}= \begin{cases}\delta_{j} & \text { if } \delta_{j}<b \\ \delta_{j}-a_{p(j)} \leq 0 & \text { if } \delta_{j} \geq b\end{cases}
$$

Now let $w=s+\sum_{i \in I \backslash I^{+}} a_{i} x_{i}$. We treat $w$ as a nonnegative continuous variable. Instead of $\bar{u}$, using $\tilde{u}$ let us write the mingling inequality (21) explicitly as

$$
w+\sum_{i \in I^{+}} b\left[x_{i}-u_{i} x(\bar{J})-\sum_{j \in J_{i} \backslash \bar{J}} \tilde{u}_{i j} x_{j}\right]+\sum_{j \in \bar{J}}\left(a_{j}+\kappa\right) x_{j}+\sum_{j \in J \backslash \bar{J}}\left(a_{j}+\sum_{i \in I_{j}} a_{i} \tilde{u}_{i j}\right)^{+} x_{j} \geq b .
$$

Using the same notation, consider also the following relaxation of the base inequality

$$
w+\sum_{i \in I^{+}} a_{i}\left[x_{i}-u_{i} x(\bar{J})-\sum_{j \in J_{i} \backslash \bar{J}} \tilde{u}_{i j} x_{j}\right]+\sum_{j \in \bar{J}}\left(a_{j}+\kappa\right) x_{j}+\sum_{j \in J \backslash \bar{J}}\left(a_{j}+\sum_{i \in I_{j}} a_{i} \tilde{u}_{i j}\right)^{+} x_{j} \geq b .
$$

Let $(\bar{x}, \bar{s})$ be a feasible point of $K_{\geq}$. We will examine two cases and show that $(\bar{x}, \bar{s})$ satisfies inequality (25). First assume that $\bar{x}(\bar{J}) \geq 1$. In this case $\left[\bar{x}_{i}-u_{i} \bar{x}(\bar{J})-\right.$ $\left.\sum_{j \in J_{i} \backslash \bar{J}} \tilde{u}_{i j} \bar{x}_{j}\right] \leq 0$ for all $i \in I^{+}$as $\bar{x}_{i} \leq u_{i}$. Therefore, inequality (25) is a relaxation of (26) for $(\bar{x}, \bar{s})$.

So, we can now assume that $\bar{x}(\bar{J})=0$. If $\bar{x}(J \backslash \bar{J}) \neq 0$, let $j^{\prime}:=\operatorname{argmin}_{j \in J}\left\{a_{j}:\right.$ $\left.\bar{x}_{j} \geq 1\right\}$ and note that $j^{\prime} \in J \backslash \bar{J}$ as $\bar{x}(\bar{J})=0$. Now let $\ell:=p\left(j^{\prime}\right)$; thus, $I_{j^{\prime}}=$ $\{1, \ldots, \ell\}$. In other words, $j^{\prime} \in J_{i}$ for all $i=1, \ldots, \ell$ and $j^{\prime} \notin J_{i}$ for any $i=$ $\ell+1, \ldots, n$. Furthermore, observe that $\tilde{u}_{i j}=u_{i}$ for $i<\ell$ and $j \in J_{i} \backslash \bar{J}$, and $\bar{x}_{j}=0$ for $j \in J_{i} \backslash \bar{J}$ for $i>\ell$. Then, for $i<\ell$, we have $\bar{x}_{i}-\sum_{j \in J_{i} \backslash \bar{J}} \tilde{u}_{i j} \bar{x}_{j} \leq \bar{x}_{i}-u_{i} \leq 0$; and for $i>\ell$, we have $\bar{x}_{i}-\sum_{j \in J_{i} \backslash \bar{J}} \tilde{u}_{i j} \bar{x}_{j}=\bar{x}_{i} \geq 0$. Thus,

$$
\bar{x}_{i}-\sum_{j \in J_{i} \backslash \bar{J}} \tilde{u}_{i j} \bar{x}_{j}\left\{\begin{array}{ll}
\leq 0 & \text { if } i<\ell \\
\geq 0 & \text { if } i>\ell
\end{array} \text { for all } i \in I^{+}\right.
$$

If $\bar{x}(J \backslash \bar{J})=0$, then let $\ell=1$ and notice that (27) still holds. As $(\bar{x}, \bar{s}) \in K_{\geq}$, it satisfies (26) and as $a_{i} \geq a_{\ell}$ for $i<\ell$ and $a_{i} \leq a_{\ell}$ for $i>\ell$ it also satisfies

$$
w+\sum_{i \in I^{+}} a_{\ell}\left[x_{i}-\sum_{j \in J_{i} \backslash \bar{J}} \tilde{u}_{i j} x_{j}\right]+\sum_{j \in J}\left(a_{j}+\sum_{i \in I_{j}} a_{i} \tilde{u}_{i j}\right)^{+} x_{j} \geq b .
$$

Therefore, it has to satisfy the $a_{\ell}$-MIR inequality for (28)

$$
w+\sum_{i \in I^{+}} b\left[x_{i}-\sum_{j \in J_{i} \backslash \bar{J}} \tilde{u}_{i j} x_{j}\right]+\sum_{j \in J}\left(a_{j}+\sum_{i \in I_{j}} a_{i} \tilde{u}_{i j}\right)^{+} x_{j} \geq b
$$

which is same as (25) when $x(\bar{J})=0$. 
We next show that the mingling inequality is facet-defining for $\operatorname{conv}\left(K_{\geq}\right)$when $I^{+}$is chosen to be $\left\{i \in I: a_{i}>b\right\}$. Furthermore, when $\bar{J} \neq \emptyset$, then it can also be facet-defining when $I^{+}$is a proper subset of $\left\{i \in I: a_{i}>b\right\}$.

Proposition 2 The mingling inequality (21) is facet-defining for $\operatorname{conv}\left(K_{\geq}\right)$if

$$
b-\min \left\{a_{j}+\kappa: j \in \bar{J}\right\} \geq \max \left\{a_{i}: a_{i}>b, i \in I \backslash I^{+}\right\} .
$$

Proof We use the notation introduced in the proof of Proposition 1. In addition, let $J=\bar{J} \cup J^{\prime} \cup J^{\prime \prime}$, where $J^{\prime}=\left\{j \in J \backslash \bar{J}: \delta_{j}<b\right\}$ and $J^{\prime \prime}=\left\{j \in J \backslash \bar{J}: \delta_{j} \geq b\right\}$, and let $I=I^{+} \cup I^{\prime} \cup I^{\prime \prime}$, where $I^{\prime}=\left\{i \in I: a_{i} \leq b\right\}$ and $I^{\prime \prime}=\left\{i \in I \backslash I^{+}: a_{i}>b\right\}$. Furthermore, let $j^{*}=\operatorname{argmin}\left\{a_{j}: j \in \bar{J}\right\}$, and note that if $\bar{J}=\emptyset$ then $I=I^{+}$and $I^{\prime \prime}=\emptyset$ by the assumption of the proposition.

It is easily seen that the following $|I|+|J|+1$ affinely independent points are on the face defined by (21):

$$
\begin{aligned}
& \begin{aligned}
\quad: \quad s=b, & \\
i \in I^{+} \quad: \quad s=0, & x_{i}=1,
\end{aligned} \\
& i \in I^{\prime} \quad: s=b-a_{i}, \quad x_{i}=1, \\
& j \in \bar{J} \quad: \quad s=b-a_{j}-\kappa, \quad x_{i}=u_{i}, i \in I^{+}, x_{j}=1, \\
& j \in J^{\prime}: s=b-\delta_{j}, \quad x_{i}=\bar{u}_{i j}, i \in I_{j}, \quad x_{j}=1, \\
& j \in J^{\prime \prime}: s=0, \quad x_{i}=\bar{u}_{i j}, i \in I_{j}, \quad x_{j}=1, \\
& i \in I^{\prime \prime} \quad: \quad s=b-a_{j^{*}}-\kappa-a_{i}, \quad x_{k}=u_{k}, k \in I^{+}, x_{j^{*}}=1, \quad x_{i}=1 \text {. }
\end{aligned}
$$

Each row above shows only the nonzero components of a point.

\subsection{Two-step mingling inequalities}

Next we will derive a second class of inequalities based on the mingling inequalities

$$
s+\sum_{i \in I^{+}} b\left(x_{i}-\sum_{j \in J_{i}} \bar{u}_{i j} x_{j}\right)+\sum_{i \in I^{+} I^{+}} a_{i} x_{i}+\sum_{j \in J} \min \left\{b, a_{j}+\sum_{i \in I_{j}} a_{i} \bar{u}_{i j}\right\} x_{j} \geq b .
$$

For any $\alpha>0$ such that $\alpha\lceil b / \alpha\rceil \leq \min _{i \in I^{+}} a_{i}$, let the two-step mingling inequality be defined as

$$
\begin{aligned}
s+\sum_{i \in I^{+}} \mu_{\alpha, b}(b)\left(x_{i}\right. & \left.-\sum_{j \in J_{i}} \bar{u}_{i j} x_{j}\right)+\sum_{i \in I^{+}} \mu_{\alpha, b}\left(a_{i}\right) x_{i} \\
& +\sum_{j \in J} \mu_{\alpha, b}\left(\min \left\{b, a_{j}+\sum_{i \in I_{j}} a_{i} \bar{u}_{i j}\right\}\right) x_{j} \geq \mu_{\alpha, b}(b),
\end{aligned}
$$

where $\mu_{\alpha, b}$ is the MIR function described in Sect. 1 . 
Observe that if $I^{+}=\emptyset$, then inequality (30) is same as the base inequality (20) and therefore inequality (31) simply becomes the $\alpha$-MIR inequality obtained from inequality (20). If $I^{+} \neq \varnothing$, however, this is not the case even though inequality (31) is obtained by applying the MIR function $\mu_{\alpha, b}$ to the coefficients of the variables in inequality (30). Notice that the terms $\left(x_{i}-\sum_{j \in J_{i}} \bar{u}_{i j} x_{j}\right), i \in I^{+}$, are not necessarily nonnegative and therefore validity of (31) does not follow from MIR. As shown in Remark 2, due to subadditivity of $\mu_{\alpha, b}$, MIR of inequality (30) produces a weaker inequality than inequality (31) for the same $\alpha$. Finally, if $b / \alpha \in \mathbb{Z}$, then inequality (30) reduces to $s \geq 0$ as does the MIR inequality (2).

Proposition 3 The two-step mingling inequality (31) is valid for $K_{\geq}$.

Proof We use the notation introduced in the proof of Proposition 1. Let $(\bar{x}, \bar{s})$ be a feasible point of $K_{\geq}$and consider the relaxation (26) of the base inequality. If $\bar{x}(\bar{J})=0$, then using the same arguments as in the proof of Proposition 1, the inequality

$$
\begin{aligned}
s+\sum_{i \in I \backslash I^{+}} a_{i} x_{i}+a_{\ell} \sum_{i \in I^{+}}\left[x_{i}-u_{i} x(\bar{J})-\sum_{j \in J_{i} \backslash \bar{J}} \tilde{u}_{i j} x_{j}\right] \\
+\sum_{j \in \bar{J}}\left(a_{j}+\kappa\right) x_{j}+\sum_{j \in J \backslash \bar{J}}\left(a_{j}+\sum_{i \in I_{j}} a_{i} \tilde{u}_{i j}\right)^{+} x_{j} \geq b
\end{aligned}
$$

is valid for $(\bar{x}, \bar{s})$ for some $\ell \in I^{+}$. Consider, again, mingling inequality (21), written in its explicit form

$$
\begin{aligned}
s+\sum_{i \in I \backslash I^{+}} a_{i} x_{i}+b \sum_{i \in I^{+}} & {\left[x_{i}-u_{i} x(\bar{J})-\sum_{j \in J_{i} \backslash \bar{J}} \tilde{u}_{i j} x_{j}\right] } \\
& +\sum_{j \in \bar{J}}\left(a_{j}+\kappa\right) x_{j}+\sum_{j \in J \backslash \bar{J}}\left(a_{j}+\sum_{i \in I_{j}} a_{i} \tilde{u}_{i j}\right)^{+} x_{j} \geq b .
\end{aligned}
$$

As both inequalities (32) and (33) are valid for $(\bar{x}, \bar{s})$, inequality

$$
\begin{aligned}
s+\sum_{i \in I \backslash I^{+}} a_{i} x_{i}+\beta \sum_{i \in I^{+}} & {\left[x_{i}-u_{i} x(\bar{J})-\sum_{j \in J_{i} \backslash \bar{J}} \tilde{u}_{i j} x_{j}\right] } \\
& +\sum_{j \in \bar{J}}\left(a_{j}+\kappa\right) x_{j}+\sum_{j \in J \backslash \bar{J}}\left(a_{j}+\sum_{i \in I_{j}} a_{i} \tilde{u}_{i j}\right)^{+} x_{j} \geq b
\end{aligned}
$$

is valid for $(\bar{x}, \bar{s})$ for any $\beta$ such that $b \leq \beta \leq a_{\ell}$. Then, choosing $b \leq \beta \leq \underline{\mathrm{a}}:=$ $\min _{i \in I^{+}} a_{i}$ ensures validity of (34) for $(\bar{x}, \bar{s})$ provided that $\bar{x}(\bar{J})=0$. 
On the other hand, if $\bar{x}(\bar{J}) \geq 1$, as $\left[\bar{x}_{i}-u_{i} \bar{x}(\bar{J})-\sum_{j \in J_{i} \backslash \bar{J}} \tilde{u}_{i j} \bar{x}_{j}\right] \leq 0$ for all $i \in I^{+}$, inequality (34) is a relaxation of (26) for $(\bar{x}, \bar{s})$ provided that $\beta \leq \underline{\text { a. }}$.

Hence, (34) is valid for all $(\bar{x}, \bar{s}) \in K_{\geq}$provided that $b \leq \beta \leq \underline{\text { a. By taking }}$ $\beta=\alpha\lceil b / \alpha\rceil$, (31) simply becomes the $\alpha$-MIR inequality for (34). Note that, $\mu_{\alpha, b}(b)=$ $\mu_{\alpha, b}(\alpha\lceil b / \alpha\rceil)$ by definition, and as $\beta$ is an integer multiple of $\alpha$, it is not necessary for the integer term $\sum_{i \in I^{+}}\left[x_{i}-u_{i} x(\bar{J})-\sum_{j \in J_{i} \backslash \bar{J}} \tilde{u}_{i j} x_{j}\right]$ in (34) to be nonnegative for the corresponding $\alpha$-MIR inequality to be valid for $K_{\geq}$(see [7]).

Remark 3 If $I^{+}=\left\{i \in I: a_{i}>b\right\}$ and $\alpha$ is chosen such that $\min _{i \in I^{+}} a_{i} \geq \alpha \geq b$, then mingling inequality (21) dominates two-step mingling inequality (31). To see this, observe that for such $\alpha$, we have $\mu_{\alpha, b}(a)=a$ for $0 \leq a \leq b$. On the other hand, $\mu_{\alpha, b}(a) \geq a$ for any $a \leq 0$ and $\alpha>0$.

Proposition 4 The two-step mingling inequality (31) is facet-defining for $\operatorname{conv}\left(K_{\geq}\right)$ if $b>0, \bar{J}=\emptyset, I^{+}=\left\{i \in I: a_{i} \geq \alpha\lceil b / \alpha\rceil\right\}$, and $\alpha=a_{i}$ for some $i \in I$.

Proof We show in Sect. 5.2 that if $I^{+}=\left\{i \in I: a_{i} \geq \alpha\lceil b / \alpha\rceil\right\}$, then two-step mingling inequalities become the continuous integer cover inequalities [1] obtained by superadditive lifting with integer variables [2]. These inequalities are facet-defining for $\operatorname{conv}\left(K_{\geq}\right)$when $b>0, \bar{J}=\emptyset$, and $\alpha=a_{i}$ for some $i \in I$ as shown in Theorem 6 of Atamtürk [1].

\section{Symmetric inequalities}

In this section we present inequalities for the mixed-integer knapsack set $K_{\geq}$when $b \leq 0$. The two classes of inequalities we present below are "symmetric" to the mingling inequality (21) and the two-step mingling inequality (31) developed for the case when $b \geq 0$.

To develop the symmetric inequalities, we use a basic observation that shows the correspondence between the facets of $\operatorname{conv}\left(K_{\geq}\right)$and the facets of $\operatorname{conv}\left(K_{\leq}\right)$, where

$$
K_{\leq}=\left\{(x, t) \in \mathbb{Z}^{N} \times \mathbb{R}: a x \leq b+t, u \geq x \geq 0, t \geq 0\right\} .
$$

Based on this observation, we utilize the results in the previous section. Note that we do not restrict the sign of $a$ or $b$ in the following lemma.

Lemma 2 Inequality $\pi x+s \geq \pi_{o}$ is valid for $K_{\geq}$if and only if inequality $(a-\pi) x \leq$ $b-\pi_{o}+t$ is valid for $K_{\leq}$. Moreover, $\pi x+s \geq \pi_{o}$ defines a facet of $\operatorname{conv}\left(K_{\geq}\right)$if and only if $(a-\pi) x \leq b-\pi_{o}+t$ defines a facet of $\operatorname{conv}\left(K_{\leq}\right)$.

Proof To see the first part, by adding a slack variable $s$ let us write $K_{\leq}$as

$$
K=\left\{(x, s, t) \in \mathbb{Z}^{N} \times \mathbb{R} \times \mathbb{R}: a x+s=b+t, u \geq x \geq 0, s, t \geq 0\right\}
$$

and consider its "relaxation" $K_{\geq}$obtained by dropping $t$. Because $\pi x+s \geq \pi_{o}$ is valid for $K_{\geq}$, it is also valid for $K$. Substituting $b+t-a x$ for $s$, we obtain 
$(a-\pi) x \leq b-\pi_{o}+t$ as a valid inequality for $K_{\leq}$. The other direction is the same. For the second part, observe that $\operatorname{conv}\left(K_{\leq}\right)$is isomorphic to $\operatorname{conv}(K)$, which is isomorphic to conv $\left(K_{\geq}\right)$. Thus, there is a one-to-one correspondence between the facets of $\operatorname{conv}\left(K_{\leq}\right)$and $\operatorname{conv}\left(K_{\geq}\right)$.

Based on this observation, we next describe how to derive valid inequalities for $K_{\geq}$when $b \leq 0$. First we multiply the base inequality $a x \geq b$ by -1 to obtain an equivalent representation of the set in $K_{\leq}$form with a nonnegative right-hand-side. We then use Lemma 2, and utilize the facets of the corresponding $K_{\geq}$set (again with a nonnegative right-hand-side) to obtain facets of the $K_{\leq}$representation. The inequalities presented below generalize the reverse continuous cover inequality developed by Marchand and Wolsey [9] and the continuous integer knapsack pack inequality of Atamtürk [1,3]. We compare them in detail later in Sect. 5.

\subsection{Symmetric mingling inequalities}

We now consider the case $b \leq 0$ for $K_{\geq}$. Our approach this time is to update the coefficients of $x_{i}, i \in I$, in the base inequality of $K_{\geq}$using the upper bounds of $x_{j}$, $j \in J$, to get a more convenient form (36). Toward this end, let $J^{-}:=\{1, \ldots, m\}$ be a nonempty subset of $\left\{j \in J: a_{j}<b\right\}$, indexed in nondecreasing order of $a_{j}$ 's, $v:=\sum_{j \in J^{-}} a_{j} u_{j}$, and $\bar{I}:=\left\{i \in I: a_{i}+v>0\right\}$. For $i \in I \backslash \bar{I}$, let

$$
J_{i}:=\{1, \ldots, p(i)\}, \text { where } p(i):=\min \left\{p \in J^{-}: a_{i}+\sum_{j=1}^{p} a_{j} u_{j} \leq 0\right\}
$$

and

$$
k_{i}:=\min \left\{k \in \mathbb{Z}_{+}: a_{i}+\sum_{j=1}^{p(i)-1} a_{j} u_{j}+a_{p(i)} k \leq 0\right\}
$$

For $i \in \bar{I}$, we let $J_{i}:=J^{-}, p(i):=m$, and $k_{i}:=u_{m}$. For and $i \in I$ and $j \in J_{i}$, let

$$
\bar{u}_{j i}:= \begin{cases}u_{j}, & \text { if } j<p(i) \\ k_{i}, & \text { if } j=p(i)\end{cases}
$$

For $j \in J$, let $I_{j}:=\left\{i \in I: j \in J_{i}\right\}$. Note $I_{j}=\varnothing$ for $j \in J \backslash J^{-}$. Using these mingling sets, the base inequality of $K_{\geq}$can be written as

$$
\sum_{j \in J} a_{j}\left(x_{j}-\sum_{i \in I_{j}} \bar{u}_{j i} x_{i}\right)+\sum_{i \in I}\left(a_{i}+\sum_{j \in J_{i}} a_{j} \bar{u}_{j i}\right) x_{i}+s \geq b .
$$


We define the symmetric mingling inequality corresponding to (36) as

$$
\sum_{j \in J^{-}}\left(a_{j}-b\right)\left(x_{j}-\sum_{i \in I_{j}} \bar{u}_{j i} x_{i}\right)+\sum_{i \in I} \min \left\{a_{i}+\sum_{j \in J_{i}} a_{j} \bar{u}_{j i}-b, 0\right\} x_{i}+s \geq 0 .
$$

Observe that if $J^{-}=\emptyset$, then inequality (37) reduces to $s \geq 0$.

Proposition 5 The symmetric mingling inequality (37) is valid for $K_{\geq}$. Furthermore, it is facet-defining for $\operatorname{conv}\left(K_{\geq}\right)$provided that

$$
\min \left\{a_{j}: a_{j}<b, j \in J \backslash J^{-}\right\} \geq \max \left\{a_{i}+v: i \in \bar{I}\right\}
$$

Proof After rewriting the base inequality of $K_{\geq}$as

$$
\sum_{j \in J}-a_{j} x_{j}+\sum_{i \in I}-a_{i} x_{i} \leq-b+s
$$

in $K_{\leq}$form, we use the corresponding $K_{\geq}$set

$$
\sum_{j \in J}-a_{j} x_{j}+\sum_{i \in I}-a_{i} x_{i}+s \geq-b
$$

to generate mingling inequalities of Sect. 3.1 as $-b \geq 0$. Using the mingling sets defined in this section, the corresponding mingling inequality (21) for (38) is

$$
\begin{aligned}
& \sum_{j \in J^{-}}-b\left(x_{j}-\sum_{i \in I_{j}} \bar{u}_{j i} x_{i}\right)+\sum_{j \in J \backslash J^{-}}-a_{j} x_{j} \\
& +\sum_{i \in I} \min \left\{-b,-a_{i}-\sum_{j \in J_{i}} a_{j} \bar{u}_{j i}\right\} x_{i}+s \geq-b .
\end{aligned}
$$

Translating this inequality for the original $K_{\leq}$set using Lemma 2, we obtain

$$
\sum_{j \in J^{-}}\left(-a_{j}+b\right) x_{j}-\sum_{j \in J^{-}} \sum_{i \in I_{j}} b \bar{u}_{j i} x_{i}+\sum_{i \in I}\left(-a_{i}-\min \left\{-b,-a_{i}-\sum_{j \in J_{i}} a_{j} \bar{u}_{j i}\right\}\right) x_{i} \leq s
$$

which is equivalent to (37). The facet condition follows from Proposition 2 and Lemma 2. 


\subsection{Symmetric two-step mingling inequalities}

In this section, we give the symmetric class of inequalities for two-step mingling inequalities for the case $b \leq 0$. For any $\alpha>0$ such that $\max _{j \in J^{-}} a_{j} \leq \alpha\lfloor b / \alpha\rfloor$, let us define the symmetric two-step mingling inequality corresponding to (36) as

$$
\begin{aligned}
& \sum_{j \in J^{-}}\left(a_{j}+\mu_{\alpha,-b}(-b)\right)\left(x_{j}-\sum_{i \in I_{j}} \bar{u}_{j i} x_{i}\right)+\sum_{j \in J \backslash J^{-}}\left(a_{j}+\mu_{\alpha,-b}\left(-a_{j}\right)\right) x_{j} \\
& +\sum_{i \in I}\left(a_{i}+\sum_{j \in J_{i}} a_{j} \bar{u}_{j i}+\mu_{\alpha,-b}\left(\min \left\{-b,-a_{i}-\sum_{j \in J_{i}} a_{j} \bar{u}_{j i}\right\}\right)\right) x_{i} \\
& +s \geq b+\mu_{\alpha,-b}(-b) .
\end{aligned}
$$

Observe that if $b / \alpha \in \mathbb{Z}$, then the symmetric two-step mingling inequality (40) reduces to the base inequality (1). Therefore, we assume below that $b / \alpha \notin \mathbb{Z}$. If $J^{-}=\varnothing$, then inequality (40) reduces to

$$
\sum_{i \in N}\left(a_{i}+\mu_{\alpha,-b}\left(-a_{i}\right)\right) x_{i}+s \geq b+\mu_{\alpha,-b}(-b),
$$

which equals the $\alpha$-MIR inequality

$$
\sum_{i \in N} \mu_{\alpha, b}\left(a_{i}\right) x_{i}+s \geq \mu_{\alpha, b}(b)
$$

because $\mu_{\alpha, b}\left(a_{i}\right)=a_{i}+\mu_{\alpha,-b}\left(-a_{i}\right)$ for $a_{i} \in \mathbb{R}$ as checked below. Let $r=b-\alpha\lfloor b / \alpha\rfloor$ and $r_{i}=a_{i}-\alpha\left\lfloor a_{i} / \alpha\right\rfloor, i \in N$. If $a_{i} / \alpha \notin \mathbb{Z}$, then

$$
\begin{aligned}
\mu_{\alpha, b}\left(a_{i}\right)-\mu_{\alpha,-b}\left(-a_{i}\right) & =r\left\lfloor a_{i} / \alpha\right\rfloor+\min \left\{r, r_{i}\right\}-(\alpha-r)\left\lfloor-a_{i} / \alpha\right\rfloor-\min \left\{\alpha-r, \alpha-r_{i}\right\} \\
& =r\left\lfloor a_{i} / \alpha\right\rfloor+\min \left\{r, r_{i}\right\}+(\alpha-r)\left\lceil a_{i} / \alpha\right\rceil-\alpha+\max \left\{r, r_{i}\right\} \\
& =\min \left\{r, r_{i}\right\}+\alpha\left\lceil a_{i} / \alpha\right\rceil-r-\alpha+\max \left\{r, r_{i}\right\} \\
& =\alpha\left\lfloor a_{i} / \alpha\right\rfloor-r+r+r_{i} \\
& =a_{i} .
\end{aligned}
$$

And if $a_{i} / \alpha \in \mathbb{Z}$, we have $\mu_{\alpha, b}\left(a_{i}\right)-\mu_{\alpha,-b}\left(-a_{i}\right)=r\left(a_{i} / \alpha\right)-(\alpha-r)\left(-a_{i} / \alpha\right)=a_{i}$.

Proposition 6 The symmetric two-step mingling inequality (40) is valid for $K_{\geq}$. Furthermore, it is facet-defining for $\operatorname{conv}\left(K_{\geq}\right)$if $b<0, \bar{I}=\emptyset, J^{-}=\left\{j \in J: a_{j} \leq\right.$ $\alpha\lfloor b / \alpha\rfloor\}$, and $\alpha=a_{j}$ for some $j \in J$. 
Proof Applying Proposition 3 to inequality (39), for any $\alpha>0$ such that $\alpha\lceil-b / \alpha\rceil \leq$ $\min _{j \in J^{-}}-a_{j}$, we obtain

$$
\begin{aligned}
& \sum_{j \in J^{-}} \mu_{\alpha,-b}(-b)\left(x_{j}-\sum_{i \in I_{j}} \bar{u}_{j i} x_{i}\right)+\sum_{j \in J \backslash J^{-}} \mu_{\alpha,-b}\left(-a_{j}\right) x_{j} \\
& +\sum_{i \in I} \mu_{\alpha,-b}\left(\min \left\{-b,-a_{i}-\sum_{j \in J_{i}} a_{j} \bar{u}_{j i}\right\}\right) x_{i}+s \geq \mu_{\alpha,-b}(-b) .
\end{aligned}
$$

Translating it to the original $K_{\leq}$form using Lemma 2 gives

$$
\begin{aligned}
& \sum_{j \in J^{-}}\left(-a_{j}-\mu_{\alpha,-b}(-b)\right) x_{j}+\sum_{j \in J \backslash J^{-}}\left(-a_{j}-\mu_{\alpha,-b}\left(-a_{j}\right)\right) x_{j}+\mu_{\alpha,-b}(-b) \sum_{j \in J^{-}} \sum_{i \in I_{j}} \bar{u}_{j i} x_{i} \\
& \quad+\sum_{i \in I}\left(-a_{i}-\mu_{\alpha,-b}\left(\min \left\{-b,-a_{i}-\sum_{j \in J_{i}} a_{j} \bar{u}_{j i}\right\}\right)\right) x_{i} \leq s-b-\mu_{\alpha,-b}(-b),
\end{aligned}
$$

which is equivalent to (40). The facet condition follows from Proposition 4 and Lemma 2.

\section{Connections with other inequalities}

In this section we present some well-known valid inequalities from the literature for knapsack sets and describe how to obtain them as (symmetric) mingling or (symmetric) two-step mingling inequalities. In particular, we consider the continuous cover and reverse continuous cover inequalities of Marchand and Wolsey [9] and the continuous integer knapsack cover and pack inequalities of Atamtürk [1,3]. We would like to emphasize that all these inequalities can be obtained by mingling when the set $I^{+}$ (or, $J^{-}$, respectively) is taken to be $\left\{i \in I: a_{i}>b\right\}$ (or, $\left\{j \in J: a_{j}<b\right\}$, respectively). When subsets of $\left\{i \in I: a_{i}>b\right\}\left(\left\{j \in J: a_{j}<b\right\}\right)$ are used for $I^{+}\left(J^{-}\right)$, the mingling procedure leads to new inequalities for these sets.

\subsection{Continuous $0-1$ cover inequalities}

Consider the mixed 0-1 knapsack set

$$
K_{\leq}^{1}:=\left\{(x, s) \in\{0,1\}^{N} \times \mathbb{R}: \sum_{i \in N} a_{i} x_{i} \leq b+s, s \geq 0\right\}
$$


where $a>0$. A subset $C$ of $N$ is called a cover if $\lambda:=\sum_{i \in C} a_{i}-b>0$. Letting $\bar{x}_{i}=1-x_{i}, i \in C$, after rewriting the base inequality as

$$
\sum_{i \in C} a_{i} \bar{x}_{i}+\sum_{i \in N \backslash C}-a_{i} x_{i}+s \geq \lambda
$$

for $I^{+} \subseteq\left\{i \in C: a_{i}>\lambda\right\}$ and $J=N \backslash C$, we obtain the mingling inequality

$$
\sum_{i \in I^{+}} \lambda\left(\bar{x}_{i}-\sum_{j \in J_{i}} x_{j}\right)+\sum_{i \in C \backslash I^{+}} a_{i} \bar{x}_{i}+\sum_{j \in N \backslash C} \min \left\{\lambda,-a_{j}+\sum_{i \in I_{j}} a_{i}\right\} x_{j}+s \geq \lambda .
$$

For $I^{+}=\left\{i \in C: a_{i}>\lambda\right\}$, mingling inequality (43) reduces to

$$
\sum_{i \in C} \min \left\{\lambda, a_{i}\right\} \bar{x}_{i}+\sum_{j \in N \backslash C}\left(-\lambda\left|I_{j}\right|+\min \left\{\lambda,-a_{j}+\sum_{i \in J_{i}} a_{i}\right\}\right) x_{j}+s \geq \lambda
$$

which is equivalent to the continuous cover inequality (Marchand and Wolsey [9]). We will illustrate inequality (44) in Example 2.

Now assume that there exists a $k \in C$ such that $\theta=a_{k}-\lambda>0$. In this case writing the base inequality as

$$
\sum_{i \in C \backslash k} a_{i} \bar{x}_{i}+\sum_{i \in N \backslash(C \backslash k)}-a_{i} x_{i}+s \geq-\theta,
$$

for $J^{-} \subseteq\left\{j \in N \backslash(C \backslash k): a_{j}>\theta\right\}$ and $I=C \backslash k$, we obtain the symmetric mingling inequality

$$
\sum_{i \in C \backslash k} \min \left\{0, a_{i}-\sum_{j \in J_{i}} a_{j}+\theta\right\} \bar{x}_{i}+\sum_{j \in J^{-}}\left(\theta-a_{j}\right)\left(x_{j}-\sum_{i \in I_{j}} \bar{x}_{i}\right)+s \geq 0 .
$$

For $J^{-}=\left\{j \in N \backslash(C \backslash k): a_{j}>\theta\right\}$, symmetric mingling inequality (46) reduces to

$$
\sum_{i \in C \backslash k}\left(\min \left\{0, a_{i}-\sum_{j \in J_{i}} a_{j}+\theta\right\}+\sum_{j \in J_{i}}\left(a_{j}-\theta\right)\right) \bar{x}_{i}-\sum_{j \in N \backslash(C \backslash k)}\left(a_{j}-\theta\right)^{+} x_{j}+s \geq 0,
$$

which is the reverse continuous cover inequality [9]. 


\subsection{Continuous integer cover inequalities}

Consider now the mixed-integer knapsack set with finite upper bounds for all integer variables

$$
K_{\leq}^{u}:=\left\{(x, s) \in \mathbb{Z}^{N} \times \mathbb{R}: \sum_{i \in N} a_{i} x_{i} \leq b+s, u \geq x \geq 0, s \geq 0\right\}
$$

where $a>0$. A subset $C$ of $N$ is called a cover if $\lambda:=\sum_{i \in C} a_{i} u_{i}-b>0$. After letting $\bar{x}_{i}=u_{i}-x_{i}, i \in C$, by rewriting the base inequality as

$$
\sum_{i \in C} a_{i} \bar{x}_{i}+\sum_{i \in N \backslash C}-a_{i} x_{i}+s \geq \lambda
$$

for $I^{+} \subseteq\left\{i \in C: a_{i}>\lambda\right\}$ and $J=N \backslash C$, we obtain the mingling inequality

$$
\begin{aligned}
& \sum_{i \in I^{+}} \lambda\left(\bar{x}_{i}-\sum_{j \in J_{i}} \bar{u}_{i j} x_{j}\right)+\sum_{i \in C \backslash I^{+}} a_{i} \bar{x}_{i}+\sum_{j \in J} \min \left\{\lambda,-a_{j}+\sum_{i \in I_{j}} a_{i} \bar{u}_{i j}\right\} x_{j} \\
& \quad+s \geq \lambda .
\end{aligned}
$$

Now assume that there exists a $k \in C$ such that $\theta=a_{k} u_{k}-\lambda>0$ and $\lambda / a_{k} \notin \mathbb{Z}$. Furthermore, let $\eta=\left\lceil\theta / a_{k}\right\rceil$ and $\rho=\theta-a_{k}\left\lfloor\theta / a_{k}\right\rfloor$. Then $\left\lceil\lambda / a_{k}\right\rceil=u_{k}-\eta+1$ and $\lambda-a_{k}\left\lfloor\lambda / a_{k}\right\rfloor=a_{k}-\rho$. For $I^{+} \subseteq\left\{i \in C: a_{i} \geq a_{k}\left\lceil\lambda / a_{k}\right\rceil\right\}$, the corresponding two-step inequality for (48) with $\alpha=a_{k}$ is then

$$
\begin{aligned}
& \sum_{i \in I^{+}}\left(u_{k}-\eta+1\right)\left(a_{k}-\rho\right)\left(\bar{x}_{i}-\sum_{j \in J_{i}} \bar{u}_{i j} x_{j}\right)+\sum_{i \in C \backslash I^{+}} \mu_{a_{k}, \lambda}\left(a_{i}\right) \bar{x}_{i} \\
& +\sum_{j \in J} \mu_{a_{k}, \lambda}\left(\min \left\{\lambda,-a_{j}+\sum_{i \in I_{j}} a_{i} \bar{u}_{i j}\right\}\right) x_{j}+s \geq\left(u_{k}-\eta+1\right)\left(a_{k}-\rho\right) .
\end{aligned}
$$

Observe that if $u_{k}=1$, then we have $a_{k}-\rho=\lambda$ and $\eta=1$. In this case, inequalities (49) and (50) are the same if $C \backslash I^{+}=\left\{i \in C: a_{i} \leq \lambda\right\}$ and $\bar{J}=\varnothing$ because $\bar{J}=\varnothing$ implies that $-a_{j}+\sum_{i \in I_{j}} a_{i} \bar{u}_{i j} \geq 0$ for all $j \in J$ and $\mu_{a_{k}, \lambda}(a)=a$ for $0 \leq a \leq \lambda<a_{k}$.

Alternatively, writing the base inequality as

$$
\sum_{i \in C \backslash k} a_{i} \bar{x}_{i}+\sum_{i \in N \backslash(C \backslash k)}-a_{i} x_{i}+s \geq-\theta,
$$


for $J^{-} \subseteq\left\{j \in N \backslash(C \backslash k): a_{j}>\theta\right\}$ and $I=C \backslash k$, we obtain the symmetric mingling inequality

$$
\sum_{i \in C \backslash k} \min \left\{0, a_{i}-\sum_{j \in J_{i}} a_{j} \bar{u}_{j i}+\theta\right\} \bar{x}_{i}+\sum_{j \in J^{-}}\left(\theta-a_{j}\right)\left(x_{j}-\sum_{i \in I_{j}} \bar{u}_{j i} \bar{x}_{i}\right)+s \geq 0 .
$$

For $J^{-} \subseteq\left\{j \in N \backslash(C \backslash k): a_{j} \geq a_{k}\left\lceil\theta / a_{k}\right\rceil\right\}$, the corresponding symmetric two-step inequality for (51) with $\alpha=a_{k}$ is then

$$
\begin{aligned}
& \sum_{j \in J^{-}}\left(-a_{j}+\eta \rho\right)\left(x_{j}-\sum_{i \in I_{j}} \bar{u}_{j i} \bar{x}_{i}\right)+\sum_{j \in J \backslash J^{-}}\left(-a_{j}+\mu_{a_{k}, \theta}\left(a_{j}\right)\right) x_{j} \\
& +\sum_{i \in C \backslash k}\left(a_{i}+\sum_{j \in J_{i}} \bar{u}_{j i}+\mu_{a_{k}, \theta}\left(\min \left\{\theta,-a_{i}+\sum_{j \in J_{i}} a_{j} \bar{u}_{j i}\right\}\right)\right) \bar{x}_{i}+s \geq-\theta+\eta \rho .
\end{aligned}
$$

Observe that if $u_{k}=1$, then we have $\rho=\theta$ and $\eta=1$. In this case, inequalities (52) and (53) are the same if $J \backslash J^{-}=\left\{j \in N \backslash(C \backslash k): a_{j} \leq \theta\right\}$ and $\bar{I}=\varnothing$ because $\bar{I}=\emptyset$ implies that $-a_{i}+\sum_{j \in J_{i}} a_{j} \bar{u}_{j i} \geq 0$ for all $i \in I=C \backslash k$ and $\mu_{a_{k}, \theta}(a)=a$ for $0 \leq a \leq \theta<a_{k}$.

For a cover $C$, Atamtürk [1,3] gives the following continuous integer knapsack cover and pack inequalities

$$
\sum_{i \in C}-\Phi_{k}\left(-a_{i}\right) \bar{x}_{i}+\sum_{j \in J}-\gamma_{k}\left(a_{j}\right) x_{j}+s \geq\left(u_{k}-\eta+1\right)\left(a_{k}-\rho\right)
$$

and

$$
\sum_{j \in J}-\Phi_{k}\left(a_{j}\right) x_{j}+\sum_{i \in C \backslash k}-\omega_{k}\left(-a_{i}\right) \bar{x}_{i}+s \geq-\theta+\eta \rho
$$

where

$$
\Phi_{k}(a)= \begin{cases}\left(\eta-u_{k}-1\right)\left(a_{k}-\rho\right) & \text { if } a<-\lambda, \\ a-\mu_{a_{k}, b}(a) & \text { if }-\lambda \leq a \leq \theta, \text { for } k \in N \\ a-\eta \rho & \text { if } a>\theta,\end{cases}
$$

and $\gamma_{k}$ and $\omega_{k}$ are superadditive lifting functions [2] described explicitly in these references. By inspection, it can be verified that if $I^{+}=\left\{i \in C: a_{i} \geq a_{k}\left\lceil\lambda / a_{k}\right\rceil\right\}$, then

$\gamma_{k}\left(a_{j}\right)=\sum_{i \in I_{j}} \bar{u}_{i j}\left(u_{k}-\eta+1\right)\left(a_{k}-\rho\right)-\mu_{\alpha, \lambda}\left(\min \left\{\lambda,\left(-a_{j}+\sum_{i \in I_{j}} a_{i} \bar{u}_{i j}\right)\right\}\right)$ for $j \in J$ 
and if $J^{-}=\left\{j \in N \backslash(C \backslash k): a_{j} \geq a_{k}\left\lceil\theta / a_{k}\right\rceil\right\}$, then

$\omega_{k}\left(a_{i}\right)=a_{i}+\sum_{j \in J_{i}} \bar{u}_{j i}(\eta \rho-1)-\mu_{\alpha, \theta}\left(\min \left\{\theta,\left(a_{i}+\sum_{j \in J_{i}} a_{j} \bar{u}_{j i}\right)\right\}\right)$ for $i \in C \backslash k$.

Hence, inequalities (50) and (53) are equivalent to (54) and (55), respectively.

\subsection{C-MIR inequalities}

As mentioned in the Introduction, complemented MIR inequalities, given by Marchand and Wolsey [10], have been successfully implemented as cutting planes in commercial MIP solvers. These cuts involve obtaining a base inequality from the MIP via constraint aggregation and then complementing some of the variables that have finite upper bounds. An $\alpha$-MIR inequality is then written for the complemented base inequality.

Here we apply the C-MIR inequalities to the mixed-integer knapsack set $K_{\leq}^{u}$ and compare them with the mingling inequalities. Let $C \subseteq N$ be a cover such that $\bar{a}:=$ $\max _{i \in C} a_{i}>\lambda$. Complementing $x_{i}, i \in C$, we can then write the complemented base inequality as

$$
\sum_{i \in C} a_{i} \bar{x}_{i}+\sum_{i \in N \backslash C}-a_{i} x_{i}+s \geq \lambda
$$

where $\bar{x}_{i}$ denotes $u-x_{i}$ as before. Consider the $\bar{a}$-MIR inequality for (56)

$$
\sum_{i \in C} \min \left\{\lambda, a_{i}\right\} \bar{x}_{i}+\sum_{i \in N \backslash C} \mu_{\bar{a}, \lambda}\left(-a_{i}\right) x_{i}+s \geq \lambda
$$

We now show that the mingling inequality (49)

$$
\sum_{i \in I^{+}} \lambda\left(\bar{x}_{i}-\sum_{j \in J_{i}} \bar{u}_{i j} x_{j}\right)+\sum_{i \in C \backslash I^{+}} a_{i} \bar{x}_{i}+\sum_{j \in J} \min \left\{\lambda, a_{j}+\sum_{i \in I_{j}} a_{i} \bar{u}_{i j}\right\} x_{j}+s \geq \lambda
$$

where $I^{+}=\left\{i \in C: a_{i}>\lambda\right\}$ and $J=N \backslash C$, dominates (57). By definition of $I^{+}$ the coefficients for $C$ are the same for both inequalities (57) and (58). For $j \in N \backslash C$, let $k_{j}=\left\lceil a_{j} / \bar{a}\right\rceil$. Then

$$
\mu_{\bar{a}, \lambda}\left(-a_{j}\right)=-\lambda k_{j}+\min \left\{\lambda, \bar{a} k_{j}-a_{j}\right\}
$$


On the other hand, the coefficient of $x_{j}, j \in J$, in (58) is

$$
\sigma_{j}:=-\lambda \sum_{i \in I_{j}} \bar{u}_{i j}+\min \left\{\lambda, \sum_{i \in I_{j}} a_{i} \bar{u}_{i j}-a_{j}\right\}
$$

We consider three cases: 1 . if $\sum_{i \in I_{j}} \bar{u}_{i j}=k_{j}$, then $\sigma_{j} \leq \mu_{\bar{a}, \lambda}\left(-a_{j}\right)$ as $\sum_{i \in I_{j}} a_{i} \bar{u}_{i j} \leq$ $\bar{a} k_{j} ; 2$. if $\sum_{i \in I_{j}} \bar{u}_{i j} \geq k_{j}+1$, then $\sigma_{j} \leq \mu_{\bar{a}, \lambda}\left(-a_{j}\right)$ as $\min \left\{\lambda,-\sum_{i \in I_{j}} a_{i} \bar{u}_{i j}-a_{j}\right\}-$ $\min \left\{\lambda, \bar{a} k_{j}-a_{j}\right\} \leq \lambda$; and finally, 3. if $\sum_{i \in I_{j}} \bar{u}_{i j} \leq k_{j}-1$, then

$$
\sigma_{j} \leq \sum_{i \in I_{j}}\left(a_{i}-\lambda\right) \bar{u}_{i j}-a_{j} \leq \sum_{i \in I_{j}}(\bar{a}-\lambda) \bar{u}_{i j}-a_{j} \leq(\bar{a}-\lambda)\left(k_{j}-1\right)-a_{j} \leq \mu_{\bar{a}, \lambda}\left(-a_{j}\right) .
$$

If $C$ is chosen to be a minimal $0-1$ cover, (57) is the familiar 0-1 knapsack cover inequality

$$
\sum_{i \in C} \lambda x_{i}+\sum_{i \in N \backslash C}-\mu_{\bar{a}, \lambda}\left(-a_{i}\right) x_{i} \leq \lambda(|C|-1)+s
$$

lifted using the MIR function $\mu_{\bar{a}, \lambda}$. The numerical example below illustrates the coefficients of $x_{i}, i \in N \backslash C$ for (61) and (43) for comparison.

Example 2 Consider a mixed 0-1 knapsack set given by

$$
13 x_{1}+10 x_{2}+9 x_{3}+8 x_{4}+5 x_{5}+a x_{6} \leq 42+s, \quad x \in\{0,1\}^{6}, s \geq 0 .
$$

For cover $C=\{1,2,3,4,5\}$, we have $\lambda=3$ and $\bar{a}=13$. Writing the knapsack inequality as

$$
\sum_{i=1}^{5} a_{i}\left(1-x_{i}\right)-a x_{6}+s \geq \lambda
$$

we see that the corresponding complemented $\bar{a}$-MIR inequality (61) is

$$
\sum_{i=1}^{5} \lambda x_{i}-\mu_{13, \lambda}(-a) x_{6} \leq 4 \lambda+s
$$

For the base inequality (62), we have $I^{+}=\{1,2,3,4,5\}$. The mingling set for $x_{6}$ is a function of its coefficient $a$. For instance, if $32<a \leq 40$, then $I_{6}=\{1,2,3,4\}$, $J_{i}=\{6\}$ for $i=1, \ldots, 4$, and $J_{5}=\varnothing$. Rewriting the base inequality (56) as

$$
\sum_{i=1}^{4} a_{i}\left[\left(1-x_{i}\right)-x_{6}\right]+a_{5}\left(1-x_{5}\right)+\left(-a+\sum_{i=1}^{4} a_{i}\right) x_{6}+s \geq \lambda,
$$




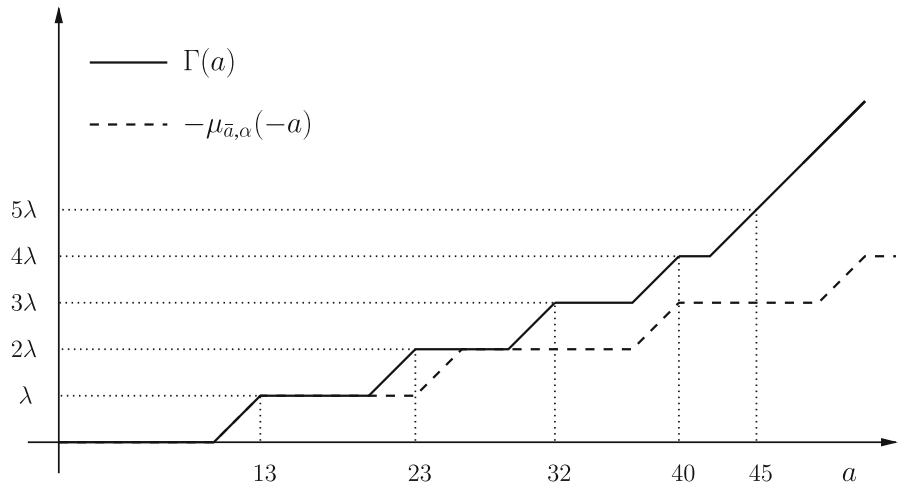

Fig. 1 Coefficients of mingling and C-MIR inequalities compared

we obtain the corresponding mingling inequality

$$
\sum_{i=1}^{4} \lambda\left[\left(1-x_{i}\right)-x_{6}\right]+\lambda\left(1-x_{5}\right)+\min \left\{\lambda,-a+\sum_{i=1}^{4} a_{i}\right\} x_{6}+s \geq \lambda
$$

or, equivalently,

$$
\sum_{i=1}^{5} \lambda x_{i}+\overbrace{\left(4 \lambda-\min \left\{\lambda,-a+\sum_{i=1}^{4} a_{i}\right\}\right)}^{\Gamma(a)} x_{6} \leq 4 \lambda+s .
$$

In Fig. 1 we plot the coefficient of $x_{6}$ as a function of $a$ for mingling and complemented MIR inequalities. In general, we have $\Gamma(a) \geq-\mu_{\bar{a}, \lambda}(-a)$ for all $a \geq 0$.

\subsection{Two-step inequalities}

We next consider the case when variables do not have finite upper bounds. In this case, let $I^{+} \subseteq\left\{i \in I: a_{i}>b\right\}, I^{+} \neq \emptyset$, and let $\bar{a}:=a_{1}=\max \left\{a_{i}: i \in I^{+}\right\}$. As $u_{i}=\infty$ for all $i \in I^{+}$, we have $\bar{J}=\emptyset$, and therefore $p(j)=1$ and $I_{j}=\{1\}$ for all $j \in J$. Furthermore, $J_{1}=J$ and $J_{i}=\emptyset$ for $i>1$. Letting

$$
k_{j}:=\bar{u}_{1 j}=-\left\lfloor a_{j} / \bar{a}\right\rfloor, \quad \text { and } \quad r_{j}:=a_{j}-\bar{a}\left\lfloor a_{j} / \bar{a}\right\rfloor, j \in J,
$$

inequality (21) becomes

$$
b\left(x_{1}+\sum_{j \in J}\left\lfloor a_{j} / \bar{a}\right\rfloor x_{j}\right)+\sum_{i \in I^{+} \backslash\{1\}} b x_{i}+\sum_{i \in I \backslash I^{+}} a_{i} x_{i}+\sum_{j \in J} \min \left\{b, r_{j}\right\} x_{j}+s \geq b,
$$


which, if $I^{+}=\left\{i \in I: a_{i}>b\right\}$ reduces to

$$
s+\sum_{j \in J}\left(\min \left\{b, r_{j}\right\}+b\left\lfloor a_{j} / \bar{a}\right\rfloor\right) x_{j}+\sum_{i \in I} \min \left\{a_{i}, b\right\} x_{i} \geq b,
$$

or, equivalently, the $\bar{a}$-MIR inequality

$$
s+\sum_{j \in J} \mu_{\bar{a}, b}\left(a_{j}\right) x_{j}+\sum_{i \in I} \mu_{\bar{a}, b}\left(a_{i}\right) x_{i} \geq \mu_{\bar{a}, b}(b)=b
$$

applied to the base inequality. In addition, for $\alpha>0$ such that $\alpha\lceil b / \alpha\rceil \leq \min \left\{a_{i}: i \in\right.$ $I^{+}$\} inequality (31) becomes

$s+\sum_{i \in I^{+}} \mu_{\alpha, b}(b) x_{i}+\sum_{i \in I \backslash I^{+}} \mu_{\alpha, b}\left(a_{i}\right) x_{i}+\sum_{j \in J}\left[\mu_{\alpha, b}\left(\min \left\{b, r_{j}\right\}\right)-\mu_{\alpha, b}(b) k_{j}\right] x_{j} \geq \mu_{\alpha, b}(b)$,

which is the two-step MIR inequality developed by Dash and Günlük [7] when applied to a base inequality $a x \geq b$ that has $\max _{i \in N}\left\{a_{i}\right\}=1>b$.

\section{Final remarks}

Mingling is a simple procedure for incorporating upper bound information into MIR cuts. The fact that many strong inequalities for the fundamental knapsack sets can also be obtained via mingling suggests that mingling may be effective as a cut generation procedure for solving general MIPs. Furthermore, because mingling uses only MIR functions to describe the cuts, mingling inequalities can be easily implemented using existing MIR routines.

We also note that (symmetric) mingling and two-step mingling inequalities can (and should) be applied after aggregating constraints of the MIP to form base inequality that defines the knapsack set. An effective approach to achieve this has been described by Marchand and Wolsey [10] for MIR inequalities.

Acknowledgments We are grateful to two anonymous referees for their valuable suggestions.

Open Access This article is distributed under the terms of the Creative Commons Attribution Noncommercial License which permits any noncommercial use, distribution, and reproduction in any medium, provided the original author(s) and source are credited.

\section{References}

1. Atamtürk, A.: On the facets of mixed-integer knapsack polyhedron. Math. Program. 98, 145-175 (2003)

2. Atamtürk, A.: Sequence independent lifting for mixed-integer programming. Oper. Res. 52, 487-490 (2004)

3. Atamtürk, A.: Cover and pack inequalities for (mixed) integer programming. Ann. Oper. Res. 139, 21-38 (2005)

4. Balas, E.: Disjunctive programming. Ann. Discret. Math. 5, 3-51 (1979) 
5. Chvátal, V.: Edmonds polytopes and a hierarchy of combinatorial problems. Discret. Math. 4, 305-337 (1973)

6. Cook, W., Kannan, R., Schrijver, A.: Chvátal closures for mixed integer programming problems. Math. Program. 47, 155-174 (1990)

7. Dash, S., Günlük, O.: Valid inequalities based on simple mixed-integer sets. Math. Program. 105, 2953 (2006)

8. Gomory, R.E.: An algorithm for the mixed integer problem. Technical Report RM-2597, The Rand Corporation (1960)

9. Marchand, H., Wolsey, L.A.: The 0-1 knapsack problem with a single continuous variable. Math. Program. 85, 15-33 (1999)

10. Marchand, H., Wolsey, L.A.: Aggregation and mixed integer rounding to solve MIPs. Oper. Res. 49, 363-371 (2001)

11. Nemhauser, G.L., Wolsey, L.A.: Integer and Combinatorial Optimization. Wiley, New York (1988)

12. Nemhauser, G.L., Wolsey, L.A.: A recursive procedure for generating all cuts for $0-1$ mixed integer programs. Math. Program. 46, 379-390 (1990) 